\title{
L'évolution récente des dépôts de matériel moteur en France
}

The recent evolution of depots of engine equipment in France

Yves Baticle

\section{OpenEdition}

\section{Journals}

Édition électronique

URL : https://journals.openedition.org/rhcf/1778

DOI : 10.4000/rhcf.1778

\section{Éditeur}

Rails \& histoire

\section{Édition imprimée}

Date de publication : 1 décembre 2003

Pagination : 275-285

ISBN : 0996-9403

ISSN : 0996-9403

\section{Référence électronique}

Yves Baticle, "L'évolution récente des dépôts de matériel moteur en France », Revue d'histoire des chemins de fer [En ligne], 28-29 | 2003, mis en ligne le 30 décembre 2014, consulté le 22 avril 2022 URL : http://journals.openedition.org/rhcf/1778 ; DOI : https://doi.org/10.4000/rhcf.1778 


\section{L'évolution récente des dépôts de matériel moteur en France}

La commission «Histoire du matériel roulant» de l'AHICF a réuni depuis 1991 une abondante documentation sur les dépôts à partir de laquelle a été publié le numéro 18 de la Revue d'histoire des chemins de fer sous le titre : «Histoire des dépôts de matériel moteur en France, 1840-1998. »

Toutefois cette documentation souffrait d'une lacune touchant en particulier la région de l'Est : le but de cet article est de combler en partie cette dernière, puis de préciser l'évolution récente du parc moteur de la SNCF de 1998 à la fin de l'année 2001.

\section{L'évolution des dépôts de l'Est (1900-1940)}

\section{Les documents}

Ceux-ci m’ont été aimablement fournis par monsieur J. Metz : ils indiquent le nom des 72 dépôts et 91 succursales du réseau et pour chacun le nombre de locomotives ainsi que celui des cheminots employés, chiffres valables pour la période 1900-1945.

\section{L'évolution}

Trois dates ont été retenues comme repères : 1900, 1920, 1940. La date de 1920 ne pose pas de difficultés malgré les destructions subies durant la Grande Guerre : la preuve en est donnée par les statistiques concernant deux dépôts situés dans la zone des combats (tabl. 1).

\begin{tabular}{|l|c|c|}
\hline & 1910 & 1920 \\
\hline Reims & 88 & 81 \\
\hline Verdun & 41 & 44 \\
\hline
\end{tabular}

Tableau 1. Evolution du nombre de machines affectées aux dépôts de Reims et de Verdun, 1910-1920. 
En revanche il a paru préférable de retenir les chiffres de 1940, plutôt que ceux de 1945 .

Le tableau et le graphique correspondant illustrent l'évolution du nombre de machines et celle du personnel pour l'ensemble des dépôts de l'Est (tabl. 2 et graphique 1).

\begin{tabular}{|l|c|c|c|}
\hline & $\mathbf{1 9 0 0}$ & $\mathbf{1 9 2 0}$ & $\mathbf{1 9 4 0}$ \\
\hline Machines & 1240 & 2445 & 2280 \\
\hline Personnel & 4830 & 12497 & 15352 \\
\hline
\end{tabular}

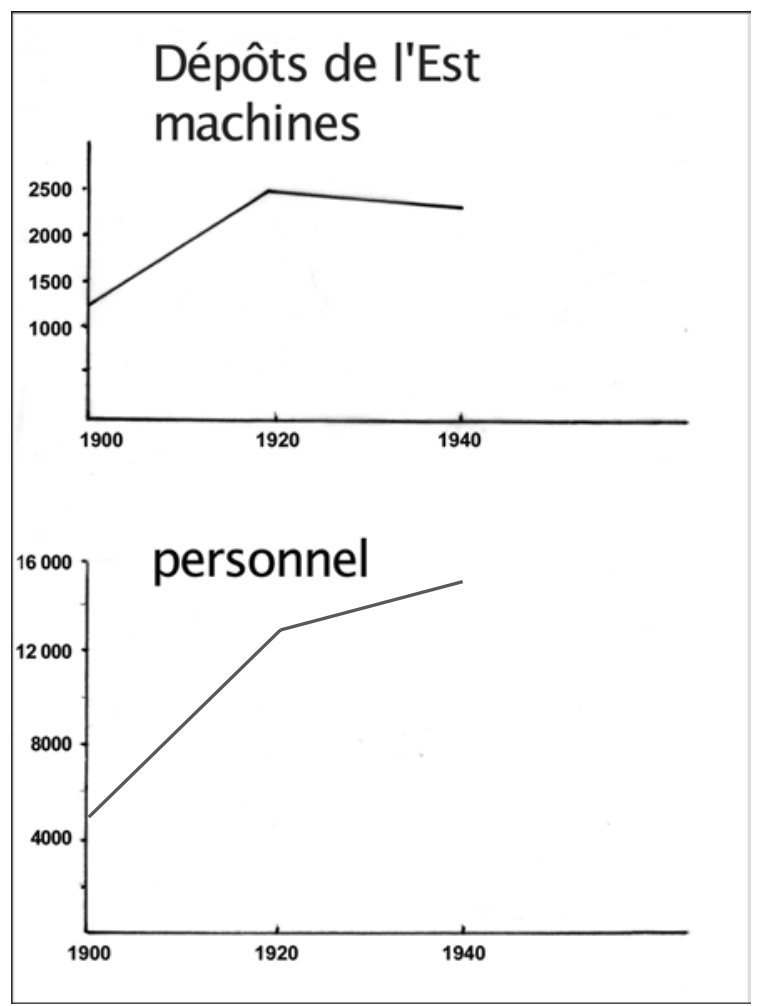

Tableau 2 et graphique 1 . Évolution des effectifs des machines et du personnel des dépôts de l'Est, 19001940.

On remarque le doublement du nombre de locomotives pendant les vingt premières années du $\mathrm{Xx}^{e}$ siècle, la traction vapeur atteignant son apogée durant la décennie 1920-1930. 
En revanche le nombre de machines diminue après 1930 ; les raisons, valables aussi pour les autres réseaux, en sont les suivantes : les débuts de la concurrence exercée par les transports routiers, surtout vis-à-vis des lignes secondaires ; la crise de 1932, responsable de la diminution du trafic marchandises ; les conséquences en sont la disparition de certaines lignes entrainant la fermeture de dépôts annexes comme Gray, Merrey, Saint-Florentin ou Pont-Saint-Vincent. Une troisième raison d'ordre technique est la mise en circulation d'autorails sur des lignes secondaires mais aussi sur la grande artère Paris-Nancy-Strasbourg.

Plus étonnante est l'évolution du personnel qui augmente fortement de 1900 à 1920, tendance qui se poursuit de 1920 à 1940, malgré la diminution du nombre de machines : l'effectif total de la main d'œuvre triple de 1900 à 1940.

Là aussi, il s'agit d'une évolution générale en France due à des mesures sociales plus qu'à la technique : à partir du $1^{\text {er }}$ mai 1920 , une loi votée en 1919 fixe à huit heures la journée de travail et accorde aux cheminots quinze jours de congé par an ; les roulements doivent donc être assurés par un plus grand nombre d'équipes.

Les tableaux et le graphique correspondant montrent quelques exemples permettant de préciser cette évolution (tabl. 3 et 4 et graphique 2).

\begin{tabular}{|l|c|c|c|}
\hline & $\mathbf{1 9 0 0}$ & $\mathbf{1 9 2 0}$ & $\mathbf{1 9 4 0}$ \\
\hline La Villette & 77 & 92 & 131 \\
\hline Mohon & 90 & 139 & 84 \\
\hline Chaumont & 89 & 145 & 92 \\
\hline
\end{tabular}

Tableau 3. Évolution de l'effectif des machines affectées aux dépôts de La Villette, Mohon, Chaumont, 1900-1940.

\begin{tabular}{|l|c|c|c|}
\hline & $\mathbf{1 9 0 0}$ & $\mathbf{1 9 2 0}$ & $\mathbf{1 9 4 0}$ \\
\hline La Villette & 367 & 715 & 771 \\
\hline Mohon & 342 & 638 & 634 \\
\hline Chaumont & 344 & 607 & 636 \\
\hline
\end{tabular}

Tableau 4. Évolution de l'effectif du personnel affecté aux dépôts de La Villette, Mohon, Chaumont, 1900-1940. 


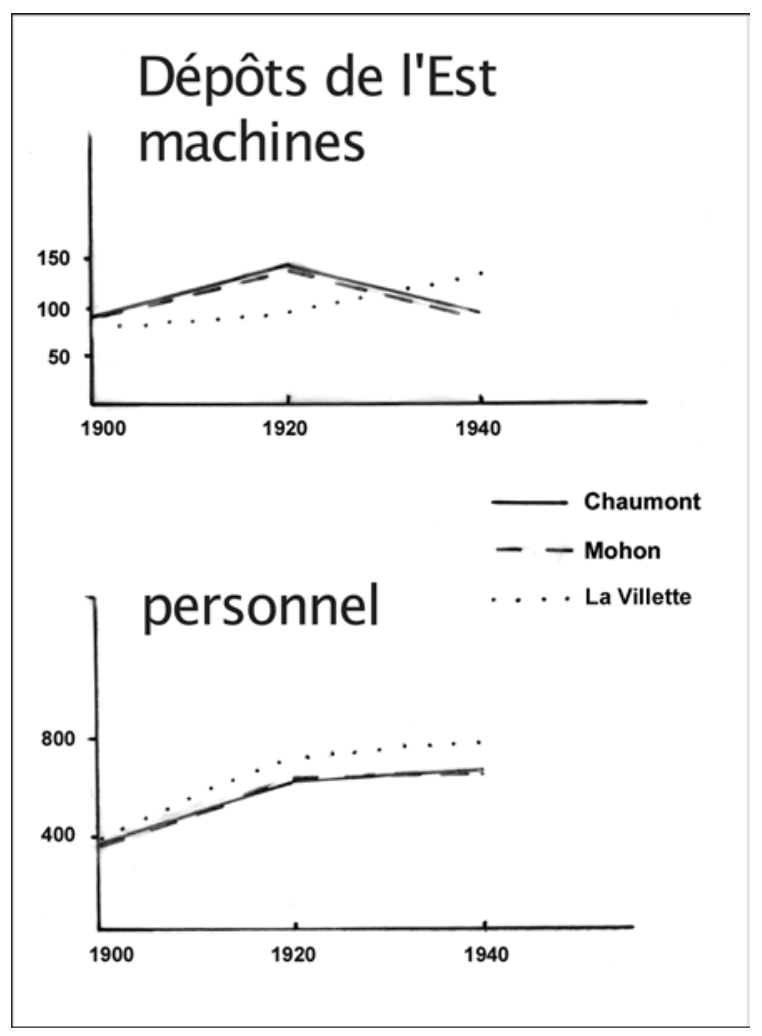

Graphique 2.

Notons tout d'abord les cas très voisins de Mohon et de Chaumont dont les coordonnées se confondent presque et sont proches de celles du graphique 1. Cette ressemblance est étonnante car ces deux dépôts ont des fonctions différentes : Mohon est un établissement concentrant des locomotives à marchandises affectées à des trains lourds sur la ligne Lille-Thionville. Chaumont est un dépôt polyvalent avec un gros parc de locomotives de vitesse desservant la ligne Paris-Belfort.

Dans les deux cas le nombre de machines s'accroît vite de 1900 à 1920, puis diminue durant les vingt années suivantes, mais pour des raisons différentes : à Mohon, crise des industries sidérurgiques à partir de 1932 ; à Chaumont, diminution du trafic voyageurs et marchandises en particulier sur les lignes secondaires d'une région à faible densité de population comme la Haute-Marne. 
Quant à la main-d'œuvre, elle suit l'évolution générale des dépôts de l'Est avec des progrès jusqu'en 1920, suivis d'une stagnation dans les vingt dernières années et même d'un léger déclin à Mohon.

Le cas du dépôt parisien de La Villette est très différent : pour les locomotives il est nettement en retrait de Mohon et Chaumont en 1900, mais il est vrai que, dès cette date, l'autre dépôt parisien, Noisyle-Sec, a déjà un effectif de 95 unités; le fait essentiel est le progrès rapide de La Villette à partir de 1920, au moment même où Mohon et Chaumont régressent.

La raison principale est l'accroissement du trafic voyageurs au départ de Paris, dû à deux faits géographiques différents : si le trafic marchandises décline, il n'en est pas de même pour les déplacements de personnes dans le sens province-Paris et vice-versa. La multiplication des rapides lourds demande l'utilisation de machines puissantes, du type 241 ; sorties en 1924, celles-ci, au nombre de quarante en 1933, sont basées à La Villette.

Le second fait est le développement très rapide, à partir des années 1930, de la banlieue le long des lignes de Gargan, Meaux et Verneuil-l'Étang; composées de dix voitures, les rames sont tractées par des locomotives-tender de type 141, lancées à partir de 1930, la plupart étant rattachée au dépôt de La Villette.

Ceci entraine évidemment l'augmentation du nombre des cheminots, roulants ou destinés à l'entretien et la réparation des machines ; l'accélération des trains de banlieue, le rythme de rotation de plus en plus élevé, demandent des changements d'équipe plus fréquents. Dès la période précédant la guerre de 1939-1945 apparait la part croissante prise par les dépôts parisiens par rapport à ceux de province.

La seconde partie de cette étude regarde l'évolution récente des dépôts français : elle ne concerne que le nombre d'engins moteurs, et que la période 1997-2001.

\section{L'évolution des dépôts de la SNCF durant les quatre dernières années (1997-2001)}

\section{Les documents}

Les effectifs sont indiqués dans les «États du matériel moteur » dressés par B. Collardey dans les numéros 15 (mai 1997) et 54 (décembre 2001) de la revue Railpassion, les données de 1997 ayant été exposées et commentées dans le numéro 18 de la Revue d'histoire des chemins de fer, déjà cité. 
Un classement pour chaque type d'engins sera tout d'abord effectué et, dans un deuxième temps, on fera la synthèse de ces résultats, afin de présenter un tableau «toutes catégories » de l'évolution des locomotives et des éléments automoteurs, une carte précisant la localisation des dépôts (fig. 1).

\section{Dépôts 2001}

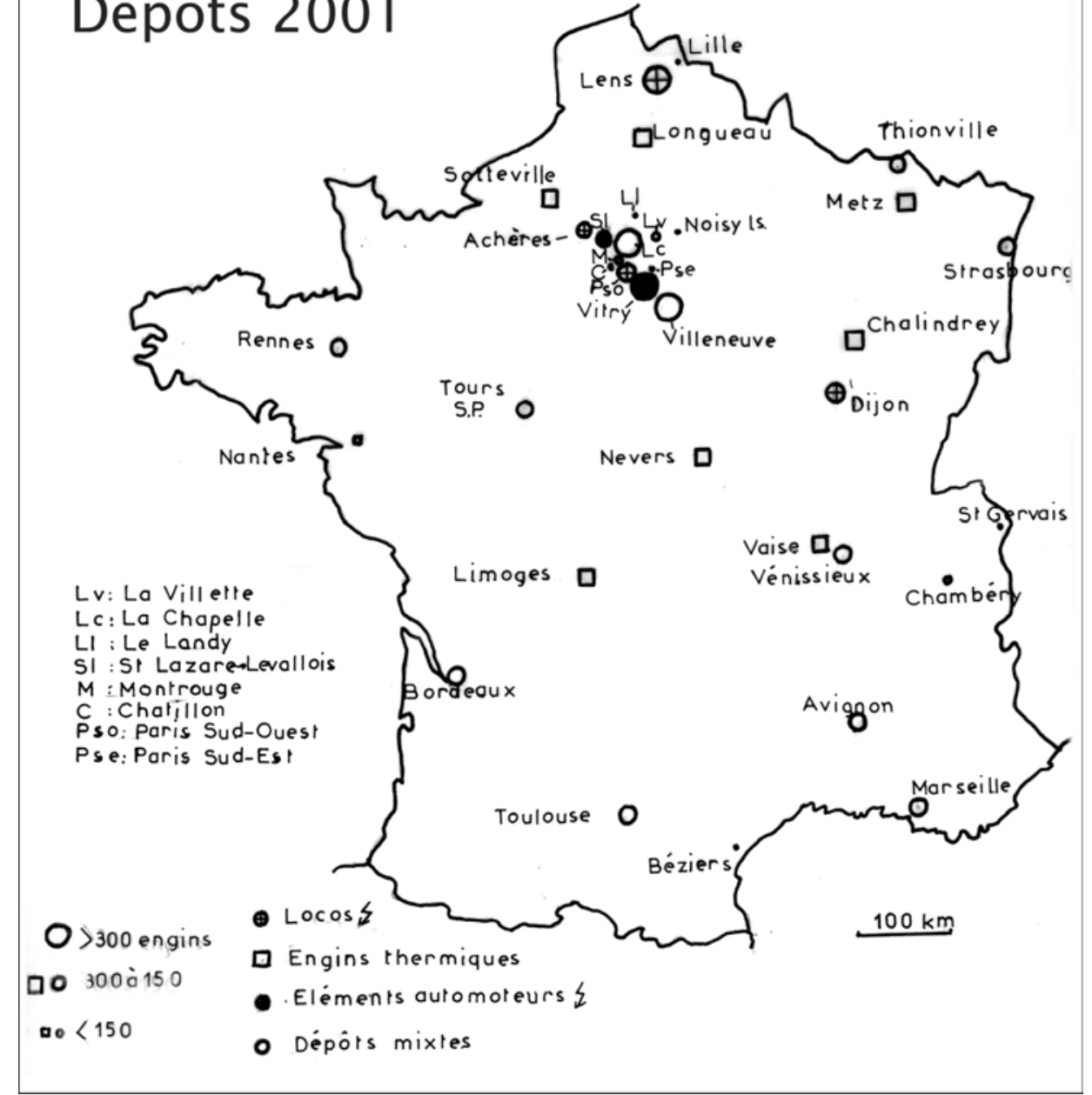

Source : Bernard Collardey, «État semestriel du matériel moteur », Rail passion, n 54 (déc. 2001), p. 94-98.

Figure 1. Les dépôts de la SNCF en novembre 2001: localisation, spécialité et effectifs des engins moteurs. ${ }^{\odot}$ Y. Baticle, 2002. 


\section{L'évolution par type d'engins}

Engins de traction à moteur thermique

\begin{tabular}{|c|c|c|}
\hline & avr-97 & nov-01 \\
\hline Nombre d'engins & 2572 & 2730 \\
\hline
\end{tabular}

Tableau 5. Évolution de l'effectif des engins de traction à moteur thermique de la SNCF, 1997-2001.

L'effectif progresse de 158 unités grâce, en particulier, aux autorails X72500 et X 73500 ; en revanche, les turbo-trains passent de 62 à 18 unités.

\begin{tabular}{|c|c|c|c|c}
\hline Longueau & Nevers & Vaise & $\begin{array}{c}\text { Avignon } \\
\text { et Tours }\end{array}$ & Sottevil \\
\hline 274 & 238 & 235 & 208 & 197 \\
\hline
\end{tabular}

Tableau 6. Répartition des engins thermiques de la SNCF par dépôt, 2001.

Ce classement a peu évolué en quatre ans ; Longueau et Nevers sont surtout des dépôts de locomotives, Vaise et Metz sont spécialisés dans les autorails. Le cas de Chalindrey est intéressant : spécialisé dans les locomotives, ce dépôt de la région Est semblait menacé en 1997 ; or, avec 167 machines, il est toujours très actif, commandant la circulation sur les axes Paris-Belfort et Dijon-Reims : un bon exemple de la vitalité de la traction thermique, dont on craignait, à tort, le déclin.

Locomotives électriques à courant continu

\begin{tabular}{|c|c|c|}
\hline & avr-97 & nov-01 \\
\hline Nombre d'engins & 838 & 678 \\
\hline
\end{tabular}

Tableau 7. Évolution de l'effectif des engins électriques ( locomotives à courant continu $-1500 \mathrm{~V}$ ) de la SNCF, avril 1997-novembre 2001.

Ces locomotives sont réparties sur dix dépôts. Depuis 1997, 160 engins ont été retirés du service, en particulier, les BB 8100, vieille série construite en 1949, et certaines BB 9200 du Sud-Ouest en cours de radiation ; en revanche, les BB 7200 font presque $30 \%$ de l'effectif. 
Le classement des dépôts montre une forte concentration sur quatre établissements, réunissant les deux tiers des machines.

\begin{tabular}{|c|c|c|r}
\hline Paris Sud-Ouest & Bordeaux & Toulouse & Vénis \\
\hline 131 & 110 & 107 & 11 \\
\hline
\end{tabular}

Tableau 8. Principaux dépôts auxquels sont affectées les locomotives à courant continu de la SNCF (1 500 V), 2001.

Avignon a perdu sa première place ; la prépondérance du SudOuest est logique, cette région ayant été électrifiée la première, dans les années 1920-1930. En revanche, les progrès de l'électrification en courant alternatif, en particulier dans le Sud-Est, expliquent que les machines à courant continu laissent de plus en plus la place à des engins bi-courant ; on comprend ainsi la quasi-disparition des engins à $1500 \mathrm{~V}$ à ToursSaint-Pierre et à Dijon.

\section{Locomotives électriques à courant alternatif et polycourants}

\begin{tabular}{|c|c|c|}
\hline & avr-97 & nov-01 \\
\hline Nombre d'engins & 1346 & 1320 \\
\hline
\end{tabular}

Tableau 9. Evolution de l'effectif des engins électriques (locomotives à courant alternatif - $25000 \mathrm{~V}$ - et polycourants) de la SNCF, avril 1997-novembre 2001.

L'effectif a baissé de 26 unités (tabl. 9) ; plus précisément, ce sont surtout les machines à courant alternatif qui ont diminué (- 98 unités), tandis que celles qui sont adaptées aux deux courants ont augmenté (+ 72 unités).

Ainsi, Lens a perdu 70 locomotives spécialisées dans la traction des trains de marchandises lourds (BB 12000 et 13 000, CC 14 000) mais a acquis la totalité des BB 36000 récemment mises en service. (tabl. 10).

Le classement des dépôts est exactement le même qu'en 1997

\begin{tabular}{|c|c|c|c|}
\hline Lens & Achères & Dijon & Strasbourg \\
\hline 241 & 201 & 199 & 130 \\
\hline
\end{tabular}

Tableau 10. Répartition des locomotives à courant alternatif et polycourants par dépôt, 2001 . 
Lens, Achères et Thionville ont un effectif équilibré entre locomotives à $25000 \mathrm{~V}$ et locomotives " polycourants » alors que Dijon et Marseille sont « polycourants » à 100 \% comme en 1997.

On constate donc un déclin normal des machines trop spécialisées au profit de celles qui peuvent rouler sur n'importe quelle ligne électrifiée.

\section{Engins automoteurs tous courants}

Dans un but de simplification sont comptés les éléments à $1500 \mathrm{~V}$, à $25000 \mathrm{~V}$, les «polycourants » et les rames desservant les deux lignes de Savoie et de Cerdagne, respectivement à 750 et $850 \mathrm{~V}$, rattachées aux EMT de Saint-Gervais et de Béziers (tabl. 11).

\begin{tabular}{|c|c|c|}
\hline & avr-97 & nov-01 \\
\hline Nombre d'engins & 1669 & 1903 \\
\hline
\end{tabular}

Tableau 11. Evolution de l'effectif des engins électriques polycourants de la SNCF, avril 1997-novembre 2001.

C'est ce type d'engins qui a le plus progressé avec 234 éléments nouveaux dont 207 rames à vitesse «normale » et 23 TGV ; l'accroissement du trafic régional dans le Nord a même justifié la création d'un EMT (Etablissement Matériel et Traction) à Lille.

Le classement des dépôts est le même qu'en 1997 (tabl. 12).

\begin{tabular}{|c|c|c|c}
\hline $\begin{array}{c}\text { Vitry- les- } \\
\text { Ardoines }\end{array}$ & La Chapelle & Villeneuve & $\mathbf{S}$ \\
\hline 382 & 314 & 173 & \\
\hline
\end{tabular}

Tableau 12. Principaux dépôts auxquels sont affectés les engins électriques polycourants, 2001.

Toutefois, il faut noter le dédoublement de certains dépôts, tous parisiens, pour assurer l'entretien et le garage des TGV (tabl. 13).

\begin{tabular}{|l|c|c|}
\hline & Nord & $\begin{array}{c}\text { Sud-Ouest et } \\
\text { Ouest }\end{array}$ \\
\hline Nombre de TGV & 105 & 105 \\
\hline Dépôt & Le Landy & Chatillon \\
\hline
\end{tabular}

Tableau 13. Répartition des rames TGV par réseau et par dépôt, 2001. 
Le total des éléments immatriculés dans l'ensemble des dépôts parisiens est impressionnant (tabl. 14).

\begin{tabular}{|c|c|c|r}
\hline Noisy-le-Sec & $\begin{array}{c}\text { La Chapelle } \\
\text { Le Landy }\end{array}$ & $\begin{array}{c}\text { Saint-Lazare } \\
\text { Chatillon }\end{array}$ & Vitr \\
\hline 130 & 419 & 268 & 382 \\
\hline
\end{tabular}

Tableau 14. Répartition des engins moteurs de la SNCF dans les dépôts d'Ile-de-France, 2001.

Le total est de 1515 éléments, soit 79,6\% des engins automoteurs français! Le premier EMT de province, Vénissieux, avec 71 éléments, vient loin derrière eux.

Cette évolution est facile à comprendre : elle est due à l'énormité du trafic de banlieue, mais aussi à la structure géographique du réseau à grande vitesse, la grande majorité des têtes de ligne étant à Paris.

\section{Le classement "toutes catégories" des dépôts}

Les deux dépôts supplémentaires sont Lille et Châtillon, compté auparavant avec celui de Montrouge. 23 ont des engins thermiques, 10 des locomotives électriques à $1500 \mathrm{~V}, 14$ des locomotives à $25000 \mathrm{~V}$ ou polycourants, 20 entretiennent des éléments automoteurs ; le total dépasse 35 puisque certains dépôts ont plusieurs spécialités : celui de Villeneuve figure dans les quatre catégories (tabl. 15).

\begin{tabular}{|l|c|c|}
\hline & $\mathbf{4 / 1 9 9 7}$ & $\mathbf{1 1 / 2 0 0 1}$ \\
\hline Engins & 6425 & 6631 \\
\hline Dépôts & 33 & 35 \\
\hline
\end{tabular}

Tableau 15. Évolution du nombre total d'engins et de dépôts de la SNCF, avril 1997novembre 2001.

\begin{tabular}{|c|c|c|r}
\hline Vitry & La Chapelle & Villeneuve & Lens \\
\hline 382 & 357 & 320 & 301 \\
\hline \hline Tours SP & Avignon & Strasbourg & Bordea \\
\hline 265 & 265 & 259 & 248 \\
\hline
\end{tabular}

Tableau 16. Classement des 10 principaux dépôts de la SNCF d'après le nombre d'engins affectés, 2001. 
Comme pour 1997, on n'a retenu que les dix premiers EMT; huit étaient déjà dans la liste en 1997 : seuls Nevers et Dijon en sont sortis, passant aux $11^{\mathrm{e}}$ et $14^{\mathrm{e}}$ places ; ils sont remplacés par ToursSaint-Pierre et Marseille.

Le fait essentiel est celui des places occupées par les dépôts parisiens : Vitry-les-Ardoines est maintenant le premier dépôt de France, tandis que Lens rétrograde de la première à la quatrième place. La raison essentielle en est le progrès des rames automotrices, concentrées dans la région Ile-de-France.

Un tiers du total des engins moteurs sont garés dans les EMT parisiens.

\section{Conclusion}

Sur le plan technique de l'organisation des dépôts, la part des éléments automoteurs par rapport à celle des locomotives s'accroît sans cesse : la conséquence essentielle est la prépondérance de la structure en gril à voies parallèles sur plusieurs centaines de mètres, aux dépens de la disposition en rotonde.

En ce qui concerne l'énergie utilisée, la traction thermique se maintient bien, surtout sur les lignes secondaires. Les locomotives électriques adaptées à un seul courant, que ce soit le $1500 \mathrm{~V}$ continu ou le $25000 \mathrm{~V}$ alternatif, ont tendance à laisser la place aux machines " polycourants ", plus rentables car adaptées à toutes les voies électrifiées.

Enfin, sur le plan géographique, grâce au développement constant du trafic de banlieue, grâce aussi au choix de Paris comme tête de lignes des réseaux TGV Nord-Atlantique et Sud-Est, les dépôts parisiens sont maintenant largement en tête du classement des EMT de la SNCF 